\title{
Spatiotemporal Analysis of Nodding Syndrome in Northern Uganda 1990-2014
}

\author{
Kizito Ongaya ${ }^{1 *}$, Augustus Aturinde ${ }^{2,4}$, Mahdi Farnaghi' ${ }^{2}$, Ali Mansourian², Gilbert Maiga1,

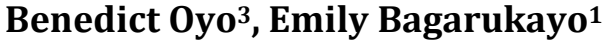

\author{
${ }^{1}$ Department of Information Systems, College of Computing and Information Sciences, Makerere University, Kampala, Uganda \\ ${ }^{2}$ GIS Center, Department of Physical Geography and Ecosystem Science, Lund University, Lund, Sweden \\ ${ }^{3}$ Department of Lands and Architectural Studies, Kyambogo University, Kampala, Uganda \\ ${ }^{4}$ Department of Computer Science, Faculty of Science, Gulu University, Gulu, Uganda \\ Email: ^kogaya@cis.mak.ac.ug, ${ }^{\star}$ ongaya.kizito@gmail.com
}

How to cite this paper: Ongaya, K., Aturinde, A., Farnaghi, M., Mansourian, A., Maiga, G., Oyo, B. and Bagarukayo, E. (2020) Spatiotemporal Analysis of Nodding Syndrome in Northern Uganda 1990-2014. Health, 12, 180-193.

https://doi.org/10.4236/health.2020.122015

Received: November 25, 2019

Accepted: February 14, 2020

Published: February 17, 2020

Copyright $\odot 2020$ by author(s) and Scientific Research Publishing Inc. This work is licensed under the Creative Commons Attribution International License (CC BY 4.0).

http://creativecommons.org/licenses/by/4.0/

\begin{abstract}
The emergence of nodding syndrome (NS) in Northern Uganda has generated controversial views with respect to patterns, natural history, and aetiology of the disease which is yet unknown. This study explored spatial patterns of NS using spatial-temporal methods to establish its clustering patterns across both space and time. Village and year of NS onset for individual patients between the years 1990 and 2014 were entered as input for spatial and temporal analysis in the 6 districts in northern Uganda where it is prevalent. Our temporal results showed that NS onset started before the population was moved in Internally Displaced People's (IDPs) camps. It also shows that NS continued to be reported during the IDPs and after people had left the IDPs. Our spatial and spatiotemporal analysis showed that two periods had persistent NS clusters. These were 2000-2004 and 2010-2014, coinciding with the period when the population was in the IDP camps and when the population was already out of the camps, respectively. Our conclusion is that the view of associating NS outbreak with living conditions in IDP camps is thus coincidental. We, therefore, contend that the actual aetiological factor of NS is still at large.
\end{abstract}

\section{Keywords}

Nodding Syndrome, Spatiotemporal, Spatial Analysis, Northern Uganda

\section{Introduction}

Nodding syndrome (NS) is a degenerative disorder that affects children originally born normal and growing well between the age of $3-18$ years [1]. It is an 
epileptic encephalopathy characterized by involuntary vertical head nodding with other types of seizures, and progressive neurological deficits [1]. The cause is unknown, yet it affected several thousand children in Africa [2]. Despite in-depth investigations by the United States Centers for Disease Control and Prevention and the Ministry of Health in Uganda, no clear causal factors have emerged [3] and no specific treatment has been found for the condition [2]. Because of the unknown pathogenesis, symptomatic treatment is the only option available for health centres to serve the affected communities [4]. The burden places upon affected communities are multifaceted; ranging from physical and mental health decline of an individual child to increasing health disparities of the entire community. It should be considered a critical and pervasive threat to human security in the affected communities of Northern Uganda because it exacerbates vulnerability to socioeconomic activities thereby results in extreme poverty to families [5].

The nodding syndrome has been reported to occur in few locations in Africa, specifically, Northern Uganda, South Sudan and Southern Tanzania [1]. In Northern Uganda, the Ministry of Health has reported that the spatial prevalence of nodding syndrome are in six districts in Northern Uganda with estimated 806 in Pader, 544 in Kitgum 339 in Lamwo, 323 in Gulu and Omoro (curved out of Gulu), 58 in Amuru, and 13 in Lira, making a total of 2143 cases to date [6].

The exact period when nodding syndrome first appeared in northern Uganda is hardly known, but plenty of documentary exist showing that the prevalence became endemic during the period when the population were in Internally Displaced People's Camps (IDPs) especially during Lord's Resistance Army (LRA) war with Government of Uganda [4] [7]. Some scholars and community members, therefore, have varying opinions about the cause and aetiology, with the local community strongly believing that the population may have been exposed to poisonous weapons, or food poison during the period when they were in the IDPs [7]. Recent scientific views have also linked NS to an abundance of blackflies, vectors that transmit the parasite that causes river blindness-onchocerciasis [1]. However, the ecology of the blackfly is widespread across the various continents thus casting scepticism on this view because NS has been identified in a few specific places mainly in East Africa only [8]. The ecology of blackflies is dynamic and influenced by environmental factors like climate, rainfall, water quality, speed of the river and much more [9].

Generally, spatial-temporal analysis and surveillance of nodding syndrome are constrained by lack of properly established aetiological (cause) factors as well as limited knowledge about environmental factors that may influence its emergence [10]. It is an illness that has eluded surveillance models in Africa for over six decades since its identification in the 1960s in South Sudan [11]. There is hardly any surveillance model for investigating spatial diffusion and supporting geographical knowledge on how to intervene on the spreading of nodding syndrome outbreak. Many authors agree that its spatial diffusion patterns and trans- 
mission models are not properly understood [1], the characteristics, risk factors, as well as aetiological factors, are also not well established [1] [10] [12] [13] complicating the surveillance efforts. Up to the year 2012, when the Ministry of Health Uganda recognized the ailment as a public health concern, it had affected estimated thousands of children in Northern Uganda [14].

The aim of this study, therefore, is to use spatial-temporal analysis techniques to investigate patterns of nodding syndrome. The study will enable us to support or question some of the views and act as a benchmark to support surveillance of NS by identifying areas of elevated NS prevalence. In the next sections, we reviewed the literature in relation to the outbreak and discussed the methodology as well as findings from our survey.

\section{Literature}

The unexplained emergence and high prevalence of NS in Northern Uganda was and still a mystery that has resulted to serious medical and socioeconomic challenges with various community views postulated to explain the cause [6] [7] [15] [16]. Despite in-depth investigations by the United States Center for Disease Control and Prevention and the Ministry of Health in Uganda, both agree that no clear causal factors have emerged [1] [4]. Apart from the unknown causal factor, the exact period when nodding syndrome prevalence hit northern Uganda is also unknown. However, first cases of NS were reported in the 1990s in Kitgum district; while response efforts by the Ministry of Health and partners towards understanding the disorder and establish management only commenced in 2009 [10]. This lead both scientific and local communities to have various views and medical anthropological postulations about the debilitating ailment, especially associating it with the Lord's Resistance Army (LRA) war that was in this part of Uganda from the early 1990s to 2004 [7]. The Ministry of Health of Uganda and partner organizations identified the gaps in knowledge of nodding syndrome that "the actual geographic coverage and distribution is not known, and that there is need for surveillance in other areas outside the current foci and the overlap of areas of distribution of nodding syndrome, etiological, potential risk factors and other information of interest [4]".

Northern Uganda experienced over two decades of war waged successively by different warlords ranging from militias of the toppled Government of Tito OkelloLutwa, Ms Alice Lakwena, OkoyaSeverino to the longest and most deadly of Lord's Resistance Army (LRA) led by Joseph Kony that confined population in Internally Displaced People's Camp (IDPs) for almost a decade [17]. The obvious views in the community are to associate the NS outbreak to the war [7]. The public opinion on the outbreak is, therefore, charged politically. In May 2012, two Members of Parliament moved a motion asking the Ugandan government to declare "Acholi sub-region" a humanitarian emergency area (PoU, 2012a). In this way, hope arose from the distribution of emergency relief after thousands of previously unhealthy children in northern Uganda affected by NS 
[7]. In addition, rumours circulated in northern Uganda about the role of government actors or chemical weapons in the aetiology of NS [7]. Further, in local discourse, feelings of political distrust are often mentioned, stretching back to a long-existing north-south divide in the country and experiences of conflict and frustration over neglect [7]. In line with the plural health beliefs most participants inhabited at least two theories of causation simultaneously. The second commonest view was that food aid distributed in the IDP camps was expired or poisoned. The maize flour had been bitter or smelt odd and often the sacks had no expiry date on them, or they did not trust that it had not been tampered with [18].

The Ministry of Health and scientific communities also have varying opinions on NS [4]. While some scholars have viewed it as a result of nutrient deficiency and poor feeding during the long period of the IDPs [19], others viewed it as associated with mouldy cereals but established no link to these divergent opinions [20]. Some have speculated that NS victims might be having malfunctioned metabolism of biotinidase and Acetyl carnitine deficiency and elevated levels of propionyl carnitine (C3) and Butyryl carnitine (C4) suggesting a possibility of a probable metabolic aetiology [21], a hypothesis not widely supported. Some scholars postulated that it is a form of autism disorder [22], a view not widely accepted too. Others studies viewed that nodding syndrome and seizures are associated with high anion gap acidosis [23].

The recent views of NS are linked to its possible association with environmental factors, mainly nematode Simulium, commonly known as blackfly, the agent that causes river blindness [19]. Research shows that blackfly carries a parasitic worm onchocerca volvulus that is responsible for causing diseases in animals and humans [1]. It was established that a high prevalence of epilepsy in various parts of Africa coincides with high prevalence of onchocerciasis [24]. Blackflies of the species Simulium Bovis normally bite cows and transmit Onchocercadukei (a cattle parasite closely related to Onchocerca volvulus), but they also bite humans, particularly those who herd cattle. Simuliumdamnosum is the main vector transmitting Onchocerca volvulus, but this also bites cows and can transmit Onchocercaochengi (another closely related cattle parasite). Humans are not known to develop any infection when exposed to the bites of blackflies infected with either Onchocercadukei or Onchocercaochengi, but they may develop antibodies against these cattle Onchocerca nematodes that may protect them against Onchocerca volvulus-related disease (or modulate the immune response in other ways) [25]. This continued inability to identify the exact causative factor provides room for numerous views and postulations to flourish.

In this study, we investigated the outbreak of NS in the light of some of these views, especially its possible association with the life of the population of northern Uganda that spent a long time in IDPs. In the following sections, we explain how data were collected from the six districts in northern Uganda, their analysis using temporal, spatial and spatiotemporal techniques, discussion and eventual conclusions are drawn. 


\section{Methodology}

The first stage of the study involved collecting data related to NS cases in northern Uganda. Then temporal analysis (using the year of NS onset) was done to evaluate the temporal trend of NS. Spatial analysis using spatial scan statistics (SaTScan) was done afterwards to understand the spatial spread of NS across the six districts of concern. Finally, spatiotemporal analysis was implemented using SaTScan (space-time) to understand the spatial-temporal trends (where and when) of NS. Details of different methodological approaches are explained below.

\subsection{Data Collection}

Ethical clearance for data collection was granted by Lacor Hospital Ethics Committee/Institution Review Board (IRB) Resenting Uganda National Council for Science and Technology (UNCST) reference number LHIREC 024/11/2016. Further authorisations were granted by the Chief Administrative Officers of the six districts under study (Amuru, Gulu, Omoro, Pader, Kitgum and Lamwo) districts. The data for twenty-four years, from 1990 to 2014, were collected from district health registries of Kitgum, Pader, Lamwo, Gulu, Amuru, and Omoro in northern Uganda. Each of these districts collected reliable data of nodding syndrome patients including date of onset and the pertaining location (County, Sub-county, Parish, and village) of each individual patient. A total of 1749 patient records were obtained. The study period 1990-2014 was chosen because these were period that represented the population of the study before, during and after the population under investigation were confined to Internally Displaced People's Camp (IDPs). Purposive selection criteria of the population under study was used. Records that shows a patient diagnosed as nodding syndrome case would meet our criteria for selection.

\subsection{Temporal Analysis}

Counts of nodding syndrome incidence were plotted against their respective years of onset and visualized using histograms. This enabled us to identify peaks and distribution of NS onset across the study period.

\subsection{Spatial Analysis}

As with the temporal analysis, the counts of nodding syndrome were aggregated to the village level for spatial visualization of the affected villages. The NS counts were further aggregated into three (3) time segments, each spanning 5 years (2000-2004, 2005-2009, and 2010-2014). The NS counts before 2000 were excluded from this spatial analysis since they were minimal. This was done through point pattern analysis. Intervals of five years were chosen because they roughly reflect the years when the affected population was in the camp, the transition time when the population was beginning to resettle back to their homes, and the period when all the population had resettled and camps decommis- 
sioned. This would help us in assessing whether the possible cause of NS was confined within the confines of the IDP camps or not.

\subsection{Spatiotemporal Analysis}

The spatiotemporal clusters within NS reported cases were detected by applying Kulldorff's spatial scan statistics [26] using SaTScan. The same technique, mostly in the spatial sense, has been widely applied in epidemiology [27] [28] [29] [30].

The basic idea of spatial scan statistics is to impose circular windows across the study area (points or polygon centroid), and then a comparison is made between the disease rate within the window and that outside the imposed window. In the spatiotemporal setting (space-time), a cylindrical window with a circular base (purely spatial) and with height corresponding to the time period of potential clusters is used. The cylindrical window moves in space and time, visiting each geographical location each possible time period. This results in an infinite number of overlapping cylinders of different sizes, jointly covering the whole study area with each cylinder representing a potential cluster. These potential clusters are evaluated through the calculation of the likelihood function (discrete Poisson) given by Equation (1) [31].

$$
L R_{x}=\left(\frac{c}{E_{[c]}}\right)^{c}\left(\frac{C-c}{C-E_{[c]}}\right)^{C-c} I\left(\frac{c}{E_{[c]}}>\frac{C-c}{C-E_{[c]}}\right)
$$

where $C$ is the total number of NS cases in the study area; $c$ is the observed number of NS cases within a window; $E_{[c]}$ is the adjusted expected number within the window under the null hypothesis; $C-E_{[c]}$ is the expected number of NS cases outside the window, and $I\left(\frac{c}{E_{[c]}}>\frac{C-c}{C-E_{[c]}}\right)$ is the binary indicator of high-risk clusters [1] or low-risk clusters [0] or both .

Important to note is the fact that the space-time permutation model uses the same function as the Poisson model. However, given the conditioning on the marginals, the observed number of cases, under the space-time settings, is only approximately Poisson distributed. The net effect of this is that the potential cluster detection procedure is no longer a formal likelihood ratio test, but it serves the same purpose as the test statistic. The obtained "likelihood ratio" values are ordered and ranked with the circle having the maximum likelihood ratio among all radius sizes at all possible centroid locations considered as the most likely cluster.

\section{Results}

The temporal incidence of nodding syndrome span from the year 1990 to 2014 . The number of cases of nodding syndrome together with the onset period (year) was visualized in a histogram given in Figure 1.

Figure 1 shows the distribution of nodding syndrome cases in Northern Uganda from 1990 to 2014. The onset started with very few cases from the 1990s 


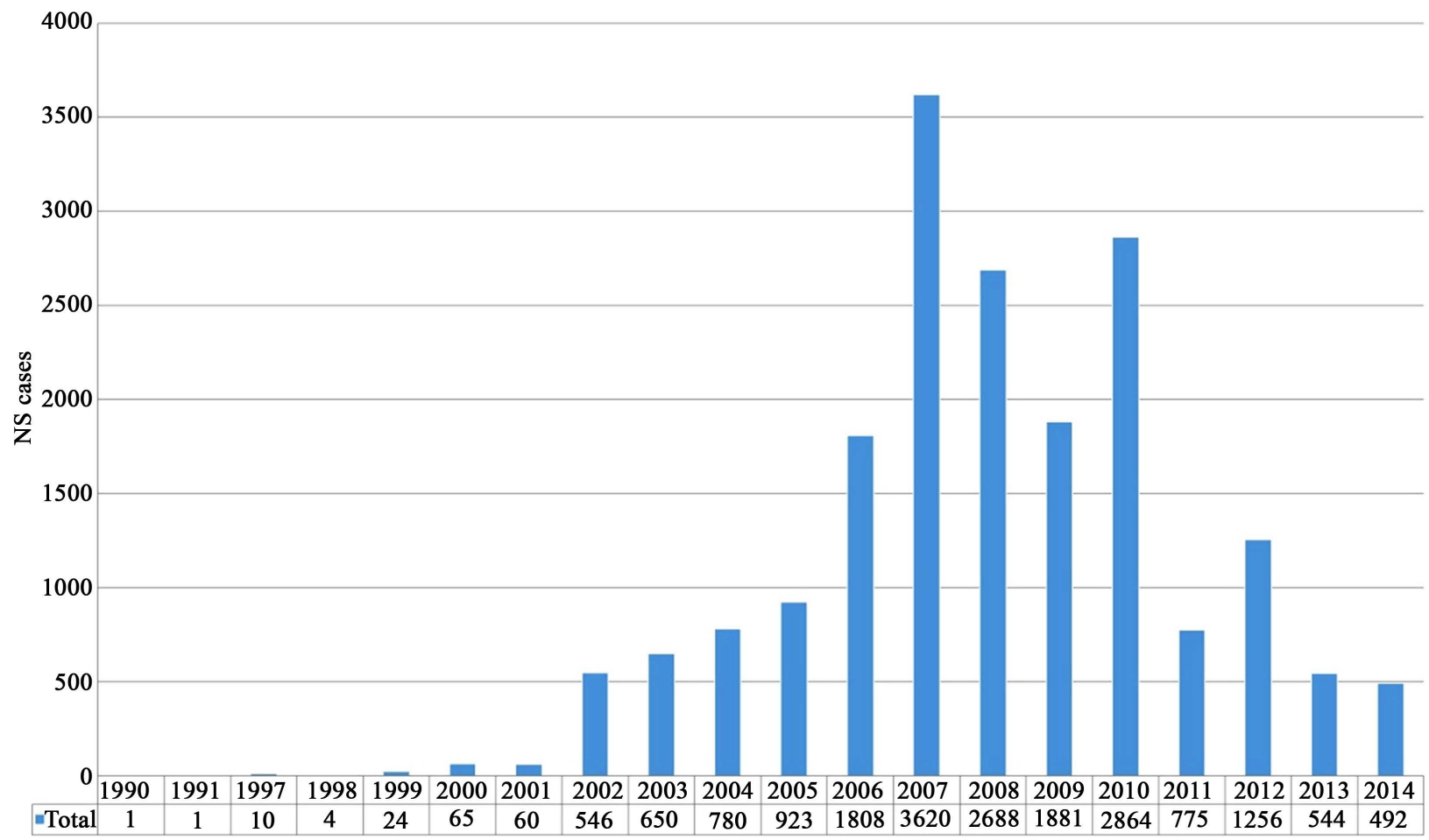

Figure 1. Nodding syndrome count against onset time in Northern Uganda in the districts of Amuru, Gulu, Omoro, Pader, Kitgum and Lamwo.

to the early 2000s. The cases increased and peaked in 2007. The number of cases remained high (albeit falling) between 2007 and 2010 before falling further to the minimum (since 2002) in 2014. It appears that conditions between 20002010 execrated onsets of nodding syndrome as war was raging in the region (see discussions-temporal incidence below).

The spatial distribution of the temporal trends in Figure 1 was not uniform across the study area. Some areas had more cases than others. To distil these differences in the spatial distribution of the NS disease burden, five-year aggregated cases were mapped at the village level, as visualized in Figure 2.

Figure 2 shows that between the years 2000-2004, most NS cases were concentrated in the areas between the northern towns of Gulu and Kitgum. During this time, these two towns had no NS reported cases. Between the years 2005-2009, the same patterns of cases being between the two towns persisted much as there some cases around Gulu and Kitgum towns. During the period 2010-2014, the reported cases were more around Kitgum town than were in other places. Generally, we notice that the cases shifted a little to the right and northwards during the 15-year study period.

Whereas the spatial analysis in Figure 2 indicated the villages that were affected and the time when this took place, it did not indicate the precise periods when the different areas experienced large enough cases to constitute a cluster. Through space-time analysis, areas of especially high incidences against their normal background incidences were highlighted and the precise periods determined. These are shown in Figure 3 and the accompanying Table 1. 


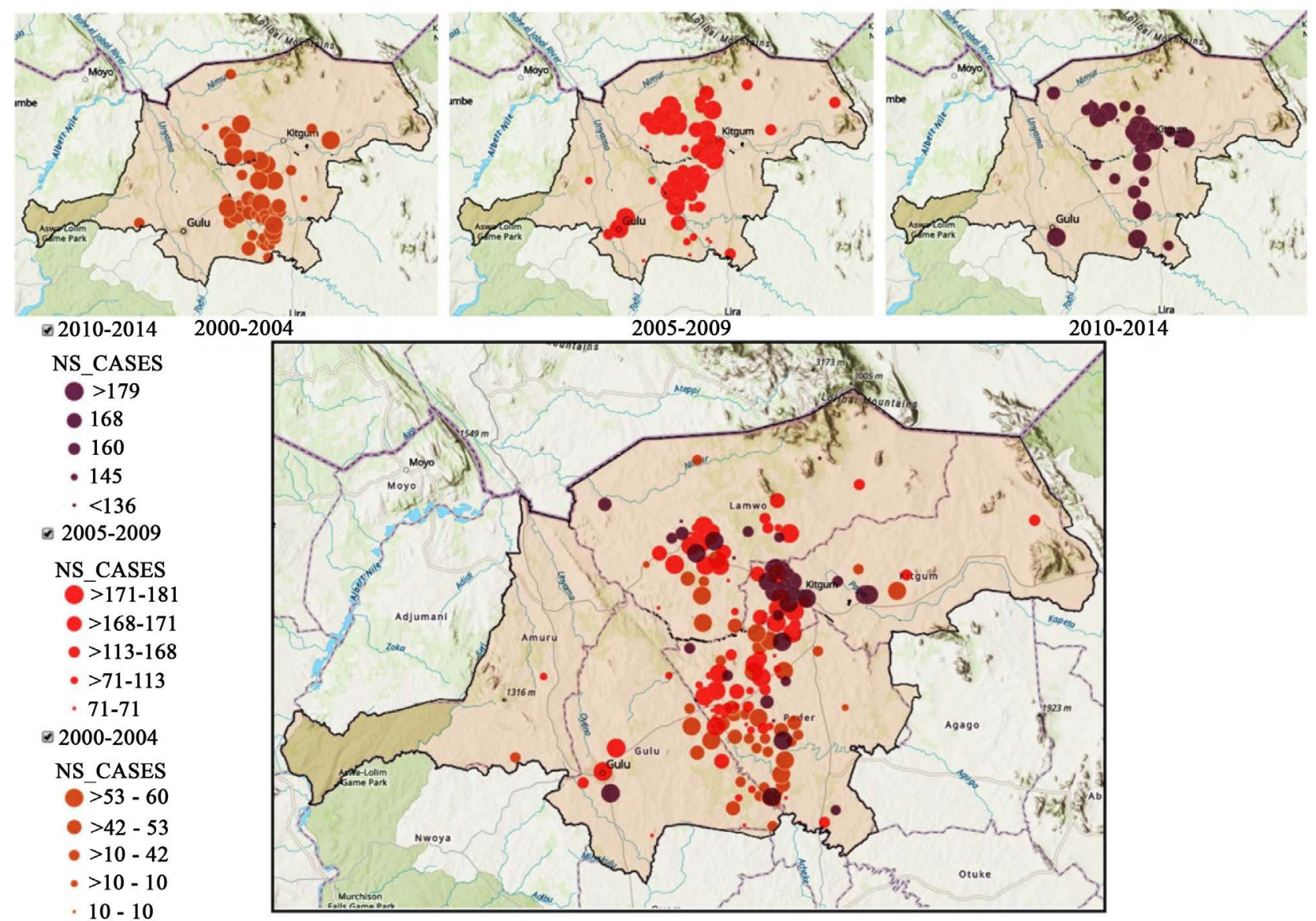

Figure 2. The aggregated spatial spread of nodding syndrome between 2000-2004, 2005-2009, 2010-2014.

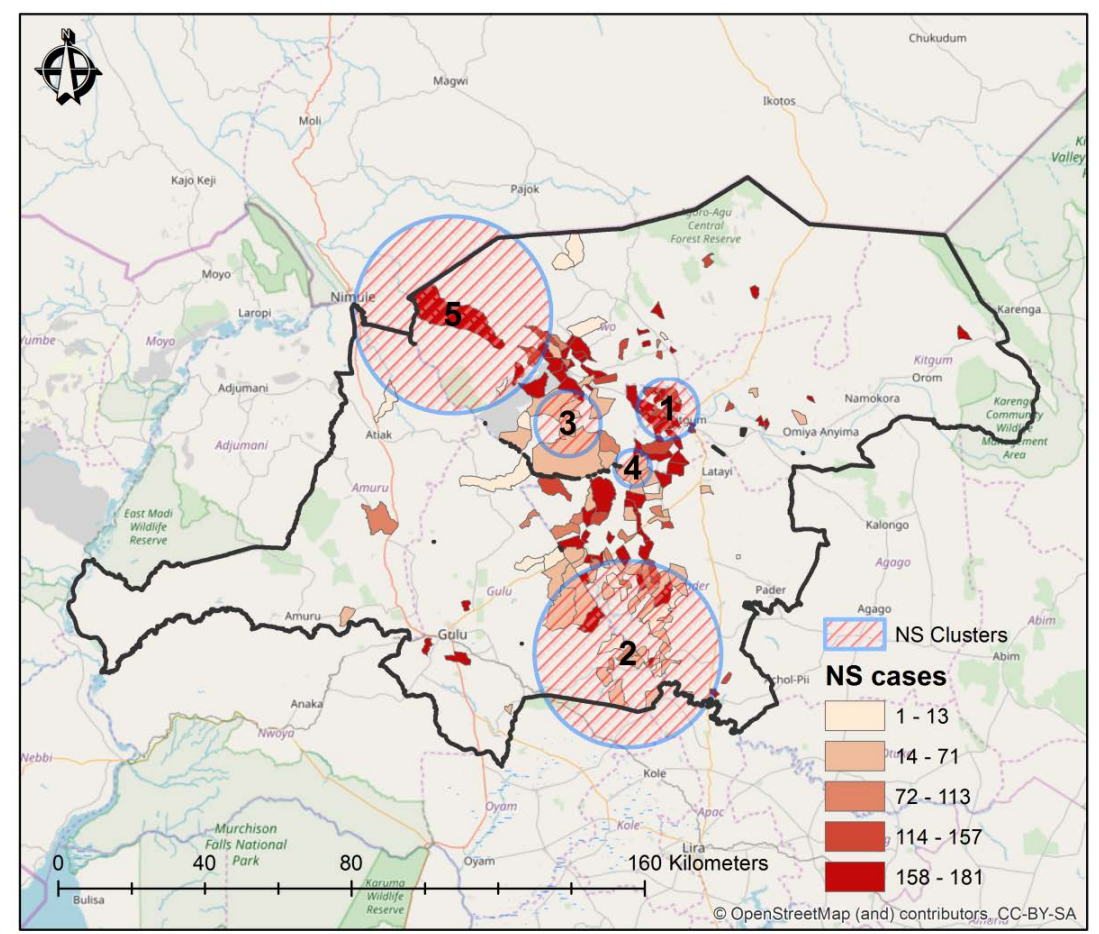

Figure 3. Space-time clusters of nodding syndrome (2000-2014). 
Figure 3 shows that there were five clusters from 2000 to 2014. Clusters 2, 3, and 4 appeared from 2000 to 2004 while clusters 1 and 5 appeared from 2010 to 2014 (shown by Table 1). Cluster 1, 3 and 4 are around Kitgum district while cluster 2 (southmost) is around Pader and Omoro districts. Cluster 5 (northmost) is in Lamwo district. No space-time clusters were observed around Gulu much as there were villages with high reported cases around it. This is also seen for other areas with high NS cases scattered across Lamwo and Kitgum.

\section{Discussions}

The discussion is based on the findings and it is structure as follows; temporal incidence of NS, spatial incidence, and spatiotemporal analysis of nodding syndrome.

\subsection{Temporal Incidence of Nodding Syndrome}

As observed in Figure 1, the NS onset started with very few cases in the first decade of the 1990s. Until 2002, the cases were still very few. From 2002 to 2007, there was a big increase in the number of reported cases. From 2007 to 2014, the cases were falling. The implication is that the aetiological agent/cause was also prevalent between 2002 to 2007 and reach some peak before ceasing to exist around 2014. Given the existing local population suspicion that there exists a link between NS and the life in the IDP camps, it is imperative to give a brief account of the period when this displacement occurred. This will enable us to chronologically discuss the incidences before and after the IDP camps.

The conflict between the LRA and the government of Uganda escalated in the mid-1990s when the LRA began receiving support from the Sudanese government. As a response to the increased rebel activities, the government embarked on a policy of forcing the population in some of the northern districts into camps to separate them from the rebels. A report from humanitarian organizations shows that the government ordered hundreds of thousands of people living in their homesteads to move to towns or trading centres within 48 hours on two occasions, in 1996 and 2002, as part of its military strategy to quell the insurgency [32]. In the period 2000 to 2004, the conflict had intensified in these northern districts, leading to new influxes of population to IDP camps [33]. The conflict had displaced up to two million people, mostly from the three Acholi districts of Gulu, Kitgum, and Pader, where roughly 90\% of the population were thought to live in camps as internally displaced persons [32].

Table 1. Clusters and their durations.

\begin{tabular}{ccc}
\hline Cluster & Start Date & End Date \\
\hline $\mathbf{1}$ & $1 / 1 / 2010$ & $12 / 31 / 2014$ \\
$\mathbf{2}$ & $1 / 1 / 2000$ & $12 / 31 / 2004$ \\
$\mathbf{3}$ & $1 / 1 / 2000$ & $12 / 31 / 2004$ \\
$\mathbf{4}$ & $1 / 1 / 2000$ & $12 / 31 / 2004$ \\
$\mathbf{5}$ & $1 / 1 / 2010$ & $12 / 31 / 2014$ \\
\hline
\end{tabular}


When we examined the temporal prevalence of NS in the early 1990s, it was very low in the community. This indicates that the cause was probably already in the community, but was not yet widespread. The NS cases increased during the period when the population was in the IDP camps (2002-2006) but remained high even when the population had left the camps (increasing to the maximum in 2007 and remaining high, although falling until 2010). The poor feeding and living conditions in the camps probably escalated their vulnerability to NS. Reports by humanitarian agencies show that the entire Acholi sub-region remained displaced, living in squalid conditions in some 200 overcrowded camps, reliant on food aid. Their traditional livelihood patterns and clan systems were destroyed and civilians, caught in the middle of the fighting between government forces and the LRA enjoyed little or no protection in or around these camps [17].

Not much research was conducted during the war period in northern Uganda. Even still, a few scholars identified that mental problems were on the rise in IDPs with expressions in the local Luo language such as "two tam/par/kumu" (depression and dysthymia-like syndromes), "ma lwor" (an anxiety-like syndrome), and a category of conduct problems referred to as "kwomaraco/gin lugero" (aggressive bad lifestyle), during this war period. The descriptions of these local syndromes are similar to Western mood, anxiety and conduct disorders, but contain some culture-specific elements [34]. Some scholars strongly believe that there is a link between IDP camp and the outbreak of NS [7] [15] [18]. These views are consistent with the period 2000-2004 when people were still in IDPs. However, findings by the Ministry of Health of Uganda in 2005, on the health status of IDPs showed that the major cause of death was malaria related with average 22 - 35 per cent of deaths in Acholi sub-region. There was no mention of mental illness (or NS) as a threat to children as well as adults [33].

Our results show that NS continued to rise beyond 2005 (Figure 1). Given that IDP camp dwellers started slowly returning to their homes by the end of 2006 [22], following the signing of the peace agreement between LRA and the Ugandan government, the continued propagation of NS beyond the period when the population were in IDPs casts doubts on whether the IDPs (and the conditions therein) were solely responsible for NS in northern Uganda. Logically, the NS cases from 2010 to 2014 are hard to attribute to conditions in the IDP camps as most families had returned to their ancestral home villages. Consequently, the search for the cause of NS should not be limited to living in the IDP camps, because children continued to fall sick many years later after the IDP camps were decommissioned. It is, therefore, possible that aetiological factor is still at large in the communities.

\subsection{Spatial Incidence of Nodding Syndrome}

Our results from Figure 2 segmented the time span into three categories of 2000-2004, 2005-2009, and 2010-2014. This was to capture the three periods of living in IDP camps (2000-2004), transition period (2005-2009) and after IDP 
camps (2010-2014). In terms of spatial spread of NS, we can note that the spatial prevalence did not change much during this time. We also note that the towns of Gulu and Kitgum towns initially had no NS cases (2000-2004). This is a surprising result as many camps were concentrated in urban centres, Kitgum and Gulu being the main ones.

In the second grouping 2005-2009, NS cases showed up in areas around the two urban centres (Gulu and Kitgum). Being a transition time when people were beginning to return to their villages, and life returning to normal, we reason that these reported cases could have been due to patients seeking better treatment at the district government hospitals. We also note that the villages most affected were in between the two towns which are associated with the network of Aswa River and its tributaries. The vector control centre identified these areas as potential breeding grounds for blackflies. In fact, there have been efforts to disinfect the fast flowing river waters of Aswa and its tributaries of the habited blackfly larvae. That notwithstanding, the association between blackflies and NS is yet to be studied from an ecological perspective, and this forms an area for further research.

\subsection{Spatiotemporal Analysis}

The spatiotemporal analysis supports what was observed in temporal and spatial analyses. The spatiotemporal clusters in 2000-2004 coincide with the period when people were confined in the IDP camps. Given that the camps were spread all over the Acholi subregion, it is not clear what can be distilled out of their (clusters 2, 3, and 4) locations (Pader, Omoro and Kitgum). The existence of Clusters 1 and 5 (Kitgum and Lamwo, respectively) from 2010 to 2014, the time when IDP camps had been long decommissioned defies the view that the IDP camps and their inherent conditions are solely to blame for the occurrence of NS in Northern Uganda.

\section{Conclusions}

Our investigation shows that the temporal onset of nodding syndrome started before the population was moved to IDPs and persisted after the population had returned to their homes. The spatial analysis shows that most villages affected by NS were located between the two northern towns of Gulu and Kitgum. Given that this coincides with the location of River Aswa and its tributaries, there could be a possible link between NS and the conditions around the river (abundance of black flies has been implicated in literature). From the spatiotemporal analysis, the two periods with clusters were 2000-2004 and 2010-2014. Whereas the life conditions around IDP camps could be linked with the 2000-2004 clusters, the existence of 2010-2014 clusters, after the decommissioning of IDP camps shows that other factors, excluding or in addition to IDP conditions, could be responsible for nodding syndrome occurrence in northern Uganda.

This research, therefore, contributes to existing knowledge in: 1) showing the time when NS was high and clustered, 2) showing the areas that were most af- 
fected, and 3) showing the time span for the established NS clusters. These three directions provide a forward step to the healthcare professionals and community in the quest for the cause of this disabling disorder of nodding syndrome.

\section{Acknowledgements}

We would like to acknowledge Makerere University for accepting to sponsor staff development of sister public universities. Particularly staff of Gulu University (Mr. OngayaKizito), staff of Kyambogo University (Mr. Atulinde Augustus). This study was funded in part by the Swedish International Development Cooperation Agency (Sida) and Makerere University under Sida contribution No: 51180060 .

We would like to acknowledge senior staff and mentors from Department of Physical Geography and Ecosystem Science, Centre for Geographic Information Systems (GIS), Lund University, Sweden and Makerere University School of Computing and Informatics Technology for spearheading the effort to implement "The Spatially Enabled E-Services Deliversity" (TSEED) Project from which this paper emanates.

This research is being carried out for academic purpose as part of doctoral program in Information Systems of Makerere University, Kampala Uganda. The authors hereby declare no conflict of interest whatsoever, in this research.

\section{Conflicts of Interest}

The authors declare no conflicts of interest regarding the publication of this paper.

\section{References}

[1] Spencer, P.S., Kitara, D.L., Gazda, S.K. and Winkler, A.S. (2016) Nodding Syndrome : 2015 International Conference Report and Gulu Accord. eNeurologicalSci, 3, 80-83. https://doi.org/10.1016/j.ensci.2015.11.001

[2] Idro, R., et al. (2019) Doxycycline for the Treatment of Nodding Syndrome (DONS); The Study Protocol of a Phase II Randomised Controlled Trial. BMC Neurology, 19, 35. https://doi.org/10.1186/s12883-019-1256-Z

[3] Van Bemmel, K. (2016) The Quest for Treatment. The Violated Body of Nodding Syndrome in Northern Uganda. Joint Polar Satellite System, 2, 63-78.

[4] Idro, R., et al. (2016) Is Nodding Syndrome an Onchocerca volvulus-Induced Neuroinflammatory Disorder? Uganda's Story of Research in Understanding the Disease. International Journal of Infectious Diseases, 45, 112-117. https://doi.org/10.1016/j.ijid.2016.03.002

[5] Lagoro, D.K. and Arony, D.A. (2017) Nodding Syndrome (NS) and Onchocerca volvulus (OV) in Northern Uganda. The Pan African Medical Journal, 28, 2-6. https://doi.org/10.11604/pamj.2017.28.1.13554

[6] Aceng, J.R. (2018) Statement on Nodding Syndrome in Northern Uganda. Ministry of Health, Uganda. https://www.health.go.ug/download/file/fid/1800

[7] van Bemmel, K. (2016) The Rise and Fall of Nodding Syndrome in Public Discourse: An Analysis of Newspaper Coverage in Uganda. Critique of Anthropology, 
36, 168-196. https://doi.org/10.1177/0308275X15614635

[8] Wotton, R.S. (1979) The Influence of a Lake on the Distribution of Blackfly Species (Diptera: Simuliidae) along a River. Oikos, 32, 368-372.

http://www.jstor.org/stable/3544

[9] Takaoka, H. (2015) 5) Review of the Biology and Ecology of Adult Blackflies in Relation to the Transmission of Onchocerciasis in Guatemala. Tropical Medicine and Health, 43, 71-85.

[10] Deogratius, M.A., David, K.L. and Christopher, O.G. (2016) The Enigmatic Nodding Syndrome Outbreak in Northern Uganda: An Analysis of the Disease Burden and National Response Strategies. Health Policy and Planning, 31, 285-292. https://doi.org/10.1093/heapol/czv056

[11] Colebunders, R. (2014) Emerging Disease in Africa. International Journal of Infectious Diseases, 21, 12. https://doi.org/10.1016/j.ijid.2014.03.429

[12] Donnelly, J. (2012) CDC Planning Trial for Mysterious Nodding Syndrome. The Lancet, 379, 299. https://doi.org/10.1016/S0140-6736(12)60126-3

[13] Burton, A. (2016) Uganda: How Goes the Nodding Syndrome War? The Lancet Neurology, 15, 30-31. https://doi.org/10.1016/S1474-4422(15)00350-6

[14] Idro, R. (2013) Nodding Syndrome; A New (Infectious?) Disease Entity of the CNS in Eastern Africa. Journal of the Neurological Sciences, 333, e51-e52. https://doi.org/10.1016/j.jns.2013.07.184

[15] Mitchell, K.B., et al. (2013) Nodding Syndrome in Northern Uganda: Overview and Community Perspectives. Epilepsy and Behavior, 26, 22-24. https://doi.org/10.1016/j.yebeh.2012.10.030

[16] Spencer, P. (2015) Nodding Syndrome: An Epileptic Disorder Restricted to Africa? Journal of the Neurological Sciences, 357, e509. https://doi.org/10.1016/j.jns.2015.09.332

[17] Finnström, S. (2006) Wars of the Past and War in the Present: The Lord's Resistance Movement/Army in Uganda. Africa, 76, 200-220. https://doi.org/10.3366/afr.2006.76.2.200

[18] Buchmann, K. (2015) 'You Sit in Fear': Understanding Perceptions of Nodding Syndrome in Post-Conflict Northern Uganda. Global Health Action, 7, Article ID: 25069.

[19] Spencer, P.S., et al. (2016) Environmental, Dietary and Case-Control Study of Nodding Syndrome in Uganda: A Post-Measles Brain Disorder Triggered by Malnutrition? Journal of the Neurological Sciences, 369, 191-203. https://doi.org/10.1016/j.jns.2016.08.023

[20] Echodu, R., et al. (2018) Is Nodding Syndrome in Northern Uganda Linked to Consumption of Mycotoxin Contaminated Food Grains? BMC Research Notes, 11, 678. https://doi.org/10.1186/s13104-018-3774-y

[21] Denis, A.A., Galloway, P., Collines, A., Frederick, M.E., Kitara, P. and David, L. (2018) Metabolic Analyses of Nodding Syndrome in Uganda: A Pilot Study Is a Biotinidase and Acetyl Carnitine Deficiency; A Metabolic Disorder. An Observational Study Design. World Journal of Pharmaceutical and Medical Research, 4, 160-174.

[22] Arony, D.A., Gazda, S. and Kitara, D.L. (2018) Could Nodding Syndrome in Northern Uganda Be a Form of Autism Spectrum Disorder? An Observational Study Design. The Pan African Medical Journal, 30, 115. https://doi.org/10.11604/pamj.2018.30.115.13634

[23] Kitara, D. (2014) High Anion Gap Metabolic Acidosis among Children with Nod- 
ding Syndrome (NS) in Northern Uganda: Case Series. British Journal of Medicine and Medical Research, 4, 1301-1314. https://doi.org/10.9734/BJMMR/2014/6151

[24] Colebunders, R., et al. (2016) Risk Factors for Epilepsy in Bas-Uélé Province, Democratic Republic of the Congo: A Case-Control Study. International Journal of Infectious Diseases, 49, 1-8. https://doi.org/10.1016/j.ijid.2016.05.018

[25] Colebunders, R., Irani, J. and Post, R. (2016) Nodding Syndrome-We Can Now Prevent It. International Journal of Infectious Diseases, 44, 61-63. https://doi.org/10.1016/j.ijid.2016.01.016

[26] Zarroug, I.M.A., et al. (2016) Seasonal Variation in Biting Rates of Simulium Damnosum Sensu Lato, Vector of Onchocerca Volvulus, in Two Sudanese Foci. PLoS ONE, 11, 1-14. https://doi.org/10.1371/journal.pone.0150309

[27] Chimoyi, L.A. and Musenge, E. (2014) Spatial Analysis of Factors Associated with HIV Infection among Young People in Uganda, 2011. BMC Public Health, 14, 555. https://doi.org/10.1186/1471-2458-14-555

[28] Smith, C.M., Lessells, R., Grant, A.D., Herbst, K. and Tanser, F. (2018) Spatial Clustering of Drug-Resistant Tuberculosis in Hlabisa Subdistrict, KwaZulu-Natal, 2011-2015. International Journal of Tuberculosis and Lung Disease, 22, 287-293. https://doi.org/10.5588/ijtld.17.0457

[29] Tiwari, N., Adhikari, C.M.S., Tewari, A. and Kandpal, V. (2006) Investigation of Geo-Spatial Hotspots for the Occurrence of Tuberculosis in Almora District, India, Using GIS and Spatial Scan Statistic. International Journal of Health Geographics, 5 , 33. https://doi.org/10.1186/1476-072X-5-33

[30] Wei, W., Wei-Sheng, Z., Ahan, A., Ci, Y., Wei-Wen, Z. and Ming-Qin, C. (2016) The Characteristics of TB Epidemic and TB/HIV Co-Infection Epidemic: A 2007-2013 Retrospective Study in Urumqi, Xinjiang Province, China. PLoS ONE, 11, e0164947. https://doi.org/10.1371/journal.pone.0164947

[31] Neill, D.B., Moore, A., Mitchell, T., Schneider, J., Cooper, G. and Lawson, A. (2006) Detection of Spatial and Spatio-Temporal Clusters. In: Handbook of Biosurveillance, Elsevier, Amsterdam, 243-254. https://doi.org/10.1016/B978-012369378-5/50018-1

[32] Norwegian Refugee Council (2005) Profile of Internal Displacement: Uganda.

[33] Ministry of Health (2005) Health and Mortality Survey among Internally Displaced Persons in Gulu, Kitgum and Pader Districts, Northern Uganda. Ministry of Health, 12-67.

[34] Betancourt, T.S., Speelman, L., Onyango, G. and Bolton, P. (2009) A Qualitative Study of Mental Health Problems among Children Displaced by War in Northern Uganda. Transcultural Psychiatry, 46, 238-256.

https://doi.org/10.1177/1363461509105815 